\title{
Computational Lexical Semantics, Incrementality, and the So-called Punctuality of Events
}

\author{
Patrick CAUDAL \\ TALANA, UFRL, Université Paris 7 \\ 2, place Jussieu \\ 75251 Paris Cedex 05, France \\ caudal@linguist.jussieu.fr
}

\begin{abstract}
The distinction between achievements and accomplishments is known to be an empirically important but subtle one. It is argued here to depend on the atomicity (rather than punctuality) of events, and to be strongly related to incrementality (i.e., to event-object mapping functions). A computational treatment of incrementality and atomicity is discussed in the paper, and a number of related empirical problems considered, notably lexical polysemy in verb - argument relationships.
\end{abstract}

\section{Introduction}

Ever since Vendler (1957) introduced it, the socalled punctuality of achievements has been the object of many theoretical contests. After having demonstrated that punctuality actually breaks up into two, distinct notions, namely non-durativity and atomicity, I will argue here for a compositional semantic account of the latter. I will show that (non-)atomicity interacts closely with the notion of incrementality, as formulated in Dowty (1991), and that this property of verbs should be lexically encoded, although it is subject both to semantics and pragmatics-driven variations. I will finally discuss the formal specifications an NLP system could use to make predictions about atomicity and incrementality.

\section{On Vendler's so-called achievements}

Vendler (1957) defined achievements and accomplishments as respectively punctual and durative. He based his claims on two main tests, noting that at <time expression> adverbials combine with achievements but not accomplishments, whereas finish combines with accomplishments but not achievements :

(1a) At what time did you reach the top? At noon sharp.

(1b) At what moment did you spot the plane? At 10:53 A.M.

(2a) *John finished leaving.

(2b) John finished drawing the circle.

Dowty (1986) and Moens and Steedman (1988) decisively questioned the coherence of the class of achievement verbs, arguing that not all of them are non-durative. As noted above, Vendler identifies punctual events through the conjunction of the (positive) at and (negative) finish tests. However, they do not always yield comparable results :

(3a) Karpov beat Kasparov at 10.00 P.M.

(3b) *The Allies beat Germany at 10.00 P.M.

(4a) *Karpov finished beating Kasparov

(4b) The Allies finished beating Germany.

The at test fails to characterize (3b) as an achievement because it is durative, whereas (3a) passes this very test because it is non-durative. On the contrary, the finish test in (4) yields an identical result for the beating of a chess player and that of a whole nation. It appears thus that the finish test does not indicate non-durativity, contrary to the at test, which refuses durative events, and that telic events such as (3b) fall outside Vendler's classification, since they fail both the finish test (unlike accomplishments) AND the at test (unlike achievements). Since it 
is desirable that achievements should include events such as (3b), durativity should not be considered as a necessary property of achievements. The salient common point between (3a) and (3b) is that both events lack proper subparts, i.e., are atomic. Atomicity should thus be regarded as the defining property of achievements; it can be tested with finish.

\section{Atomicity as a semantic issue}

Many authors, including Verkuyl (1993) and Jackendoff (1996), have denied atomicity any semantic content, and have argued that it is a pragmatic category. I do not intend to claim here that atomicity is not subject to pragmatic constraints. The following examples identify one such constraint, i.e., the relative size of arguments of verbs of consumption :

(5a) ??John finished eating the raspberry.

(5b) The bird finished eating the raspberry.

(5a) suggests that raspberries are so small with respect to a human 'eater' that eat denotes an atomic event. But the same does not hold true of birds (cf. (5b)). No attention will be paid to this kind of pragmatic constraint in this paper.

Yet I will demonstrate here that atomicity does possess a semantic content, and that therefore it can be regarded as an aspectual category. Consider the following examples ${ }^{1}$ :

(6a) *The soldier finished crossing the border.

(6b) The soldiers finished crossing the border.

(7a) *John finished slamming the door open.

(7b) John finished slamming the doors open.

The plural NPs the soldiers and the doors possess proper subparts, along which the crossing and slamming events in (6b) and (7b) are measured, making those events non-atomic (there are several distinct subevents of one door being slammed, and of one soldier crossing the border) ; compare with the atomic (6a) and (7a), where those very NPs are singular. The variation in noun quantification being a semantic one,

1 Similar examples were proposed by Declerck (1979), but were discussed in terms of durativity, and not of atomicity. atomicity should clearly receive some form of semantic content. Moreover, it should be noted that atomic events are not compatible with the progressive perfect, whereas non-atomic ones freely combine with $i^{2}$ :

$$
\begin{aligned}
& \text { *The soldier has been crossing the } \\
& \text { border. } \\
& \text { (OK with iterative, non-atomic reading) } \\
& \text { The soldiers have been crossing the } \\
& \text { border. }
\end{aligned}
$$

Those facts support a semantic view of atomicity ${ }^{3}$.

\section{Towards a semantic account : (non-) atomicity and incrementality}

The above data suggests an interesting solution to this puzzle : atomicity seems to be related to the notion of incrementality, as formulated in Dowty (1991) (see also graduality in Krifka 1992). To my knowledge, the concept of incrementality (originally proposed to account for the telicity of events) has never been discussed in the light of that of atomicity, although this is an obvious thing to do, both concepts being about the present or absence of subevents in the internal structure of events. I will undertake to bridge this gap here.

\subsection{Incrementality and delimiting arguments}

Dowty defines incrementality as a property of verbs whose development can be measured along the inner structure of one of their arguments (which he calls incremental theme) :

John drank a glass of beer.

In (9), the development of the drinking event can be measured along the subparts of the glass of beer. Each subpart of the incremental theme argument is mapped onto a subpart of the

2 Complementary tests such as the different readings of in etc. will not be studied here for want of space.

3 Caudal (1998) discusses at length related examples involving collection-referring nouns (e.g., orchestra or regiment), and shows that they behave similarly, cf. The regiment finished crossing the border. 
corresponding event (a fact which Dowty (1991) and Krifka (1992) refers to as event-object homomorphism). Dowty (1991) rejects ostensibly the possibility to treat as incremental themes the patient arguments of so-called punctual (i.e., achievement) verbs, such as slam open. According to him, incremental themes should be able to undergo a gradual change of state $^{4}$. Unfortunately, Dowty does not consider examples such as (7b), which exhibit an incremental behaviour although they include this very kind of patient argument. I will therefore reject Dowty's objection, and regard (7b) as incremental.

It follows naturally from the above definition that incrementality entails non-atomicity: it implies that a situation's development possesses proper subparts, and therefore that it is nonatomic. But does non-atomicity entail incrementality, conversely? I.e., are those two notions equivalent? If not, how should they be connected? In order to answer those questions in the following sections, I will make use of a rough feature-based notation: [+/-ATM] will express atomicity/non-atomicity, and [+/-INC] incrementality/non-incrementality.

\subsection{Non-atomicity with incrementality}

I will call delimiting arguments the arguments of a verb serving as 'measures' (or 'odometers') for the corresponding event (e.g. the internal arguments of drink or slam open). It should be noted that this term is broader than that of incremental theme, since it includes e.g., patient arguments of so-called punctual verbs, which Dowty refused to regard as incremental themes. For the sake of simplicity, I will focus in this paper exclusively on internal delimiting arguments :

(10a) John finished eating his apple.

(10b) John finished eating his apples.

(11a) *John finished throwing his stone.

(11b) John finished throwing his stones.

4 Cf. Dowty (1991:568): Many traditional Themes...are not Incremental Themes. Many achievement verbs entail a definite change of state in one of their arguments...but never in distinguishable separate stages, i.e. subevents.
(10) shows that eat can be [-ATM],[+INC] both with a definite singular and plural delimiting argument, whereas (11) shows that throw can be [-ATM],[+INC] only with a definite plural delimiting argument. The development of eating his apple is measured in (10a) along the quantity of apple remaining to eat, whereas that of throwing his stones in (11b) is measured along the successive individual stones being thrown away. I will extend the notion of incrementality to this latter kind of event-object mapping.

Under this view, incrementality arises from delimiting arguments, and not only fom incremental themes. However, I will distinguish two types of incrementality, thereby preserving a distinction between Dowty's incrementality and the extension I proposed. I will call m-incrementality (for quantity of matterincrementality) the type of incrementality exhibited by (10a) and i-incrementality (for individual-incrementality) that exhibited by (11b). At least two classes of verbs can be distinguished in this respect : verbs like eat are capable of $\mathrm{m}$-incrementality, i.e., incrementality with individual-referring delimiting arguments (they have an incremental themes in the sense of Dowty), whereas verbs like throw are only capable of i-incrementality, i.e., incrementality with collection-referring delimiting arguments (they lack an incremental theme in the sense of Dowty). Of course, non-atomicity can follow from either $i$ or $m$-incrementality.

Another type of incremental non-atomic events can be found in path-movement verbs :

\section{Mary walked the Appalachian trail.} (Tenny 1994)

The development of the walking event can be measured along the explicit path argument the Appalachian trail in (12). It is therefore [-ATM],[+INC]. White (1994) proposed a generalized path-based incremental theme role to account for the semantic behaviour of both patient and path delimiting arguments, fairly akin to the present one, since it crucially relies on a similar individual / quantity of matter distinction. One could conclude at this point that 
the present account of incrementality is sufficient to predict (non-)atomicity, and that non-atomicity and incrementality are equivalent notions. If that is right, then non-incremental events should be non-atomic. However, I will show in 3.3 that it is not the case.

\subsection{Non-atomicity without incrementality}

Some non-atomic events lack a delimiting argument, so that the type of non-atomicity involved seems unrelated to incrementality :

John finished digesting his pudding. John finished cooking the chicken. John finished registering his son at the university.

Contrary to (10) and (11b), neither (13), (14) nor (15) are (necessarily) measured along the subparts of their patient arguments. (13) and (14) are rather measured along the state of the latter, which vary as time passes. In this sense, his pudding and the chicken do not behave like delimiting arguments, and those non-atomic situations are non-incremental ([-ATM],[-INC]). Some sort of non-argumental odometer seems to be required. In the case of (13) and (14), digest and cook receive a scalar result state, i.e., one that varies as time passes: John's chicken becomes (as a whole) closer to being (finally) cooked as time passes in (14), and John's pudding gradually turns (as a whole, and not bit by bit) into nutriments inside his stomach in (13) (see Caudal $(1999 \mathrm{a} / \mathrm{b})$ for a treatment of such data). I will refer to this kind of incremental-like reading as scalarity. If one considers (15), things are somewhat different, as there exists some sort of predetermined series of stages through which one should pass in order to register at the university: John's son is closer and closer to being registered at the university as his father goes through them. I will refer to this kind of data as gradual scenarios.

I will turn now to the computational treatment of incremental non-atomic events (section 4), before suggesting some ways of accounting for non-incremental non-atomic ones (section 5).

\section{A formal, computational treatment of incremental non-atomic events}

A formal and computational treatment of incremental non-atomic events will be formulated here, relying on model-theoretic logics and on the Generative Lexicon framework (GL henceforth ; see Pustejovsky (1995) for an introduction). I will first discuss a few theoretical notions related to the internal structure of objects and events, in order to formalize $m$ and $i$-incrementality. I will leave aside the treatment of incremental patharguments, referring the interested reader to White (1994).

\subsection{Internal structure of objects and events : Link's part-of operators}

Following Link (1983), I will oppose individuals (i.e., the denotata of nouns referring to individual entities) and collections (i.e., the denotata of definite plural NPs, collectives, etc. ; see Caudal (1998a)). Let $A$ be the domain of entities (events or objects), structured as a semilattice. Let individual_part_of be a partial order relation on individual entities (henceforth $i$-part or $\leq_{\mathrm{i}}$ ), connecting an individual to the collection it belongs to. Let $U_{i}$ be the join operation on individuals and collections, $y$ a collection and $x$ an individual, such that $x$ is an $i$-part of $y$. The definition of the meronymic operator $\leq_{i}$ was formulated by Link as follows :

$$
\forall x, y\left[x \leq_{i} y \rightarrow x \sqcup_{i} y=y\right]
$$

Following again G. Link, I will define similarly a partial order relation on non-individual parts, $m$-part (or $\leq_{m}$ ), which connects an individual and its non-individual parts (e.g. a slab of stone to a rock). All those operators will apply both to events and objects in the model (events being reified). As a consequence, collection-referring NPs as well as i-incremental events are endowed with $i$-parts, whereas individual-referring NPs and $m$-incremental events possess $m$-parts.

I will argue that incrementality depends both on lexical information and structural composition. Whether events will receive (or not) an incremental reading is determined at the structural level, depending on the interaction of 
a verb with its delimiting arguments (modulo pragmatic constraints). I will now describe the lexical component of this compositional procedure.

\subsection{Encoding incrementality within the Generative Lexicon framework}

I will propose here to encode lexically whether verbs are capable of $m$-incrementality or i-incrementality. It should be noted that although the ability to exhibit m-incrementality seems to be a constant lexical property, any potentially incremental verb can receive an i-incremental reading (but recall that not all verbs can be read incrementally). In the spirit of Krifka's objectevent mapping functions (see Krifka 1992), I will assume an $i$-inc aspectual semantic role function that relates the $i$-parts of an argument to the development of an event (causing it to become $i$-incremental with an appropriate delimiting argument), and a $m$-inc function that relates the m-parts of an argument to the development of an event (causing it to become $m$-incremental with an appropriate delimiting argument). The following event/object mapping predicate MAP-I (applying only to i-inc aspectual roles) can be derived from Krifka's $\mathrm{MAP}-\mathrm{O} / \mathrm{E}$ (mapping to objects/events) predicates (see Krifka 1992:39) by replacing his standard partial order operator with $\leq_{\mathrm{i}}$ :

(17) MAP-I :

$\forall$ R[MAP-I(R) $\leftrightarrow$ MAP-E $_{i}(\mathrm{R}) \wedge$ MAP-O $\left._{i}(\mathrm{R})\right]$

$\forall$ R[MAP-E $\mathrm{E}_{\mathrm{i}}(\mathrm{R}) \leftrightarrow \forall \mathrm{E}, \mathrm{x}, \mathrm{x}^{\prime}\left[\mathrm{R}(\mathrm{e}, \mathrm{x}) \wedge \mathrm{x}^{\prime} \mathrm{S}_{\mathrm{i}} \mathrm{x} \rightarrow\right.$ $\left.\left.\exists \mathrm{e}^{\prime}\left[\mathrm{e}^{\prime} \leq_{\mathrm{i}} \mathrm{e} \wedge \mathrm{R}\left(\mathrm{e}^{\prime}, \mathrm{x}^{\prime}\right)\right]\right]\right]$

$\forall$ R[MAP-O ${ }_{i}(\mathrm{R}) \leftrightarrow \forall \mathrm{e}, \mathrm{e}^{\prime}, \mathrm{x}\left[\mathrm{R}(\mathrm{e}, \mathrm{x}) \wedge \mathrm{e}^{\prime} \leq_{\mathrm{i}} \mathrm{e} \rightarrow\right.$ $\left.\left.\exists x^{\prime}\left[x^{\prime} \leq_{i} x \wedge R\left(e^{\prime}, x^{\prime}\right)\right]\right]\right]$

A similar formulation can be given for $\mathrm{m}$-incrementality ; replace $\leq_{\mathrm{i}}$ with $\leq_{\mathrm{m}}$ in (17). Thus, by combining Link's part-of operators with Krifka's event-object mapping functions, atomicity construal functions can be formulated. Finally, GL will provide us with the proper computational lexical machinery in which to insert those functions: I will propose to encode those aspectual roles within the argument structure (ARGSTR) feature in GL, by making them bear directly on the relevant argument position. The following entries for eat and throw illustrate such an encoding for internal arguments (again, external arguments are left aside for the sake of simplicity) :

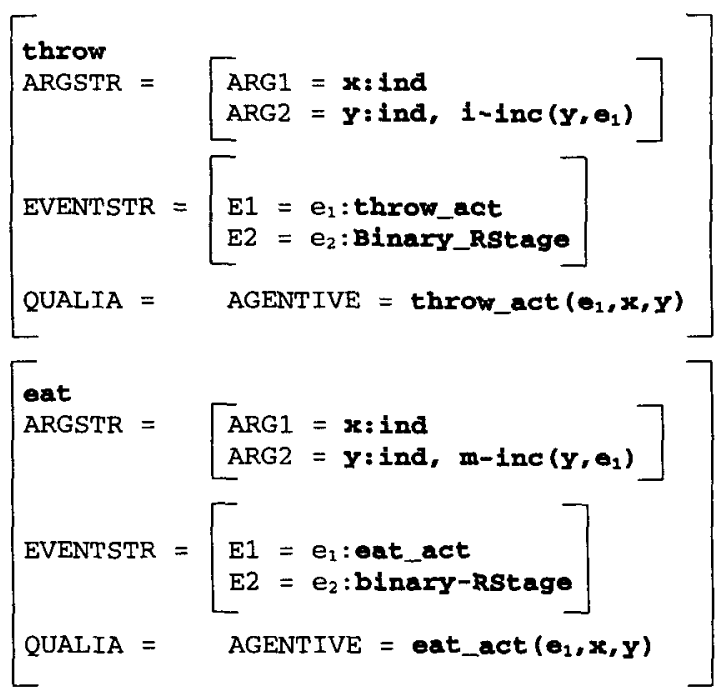

$m / i-i n c(x, e)$ indicates that the internal structures of subevent $a$ and argument $x$ are related by an homorphic mapping. If $\mathbf{x}$ possesses proper subparts, then will be incremental ; the whole point remains that incrementality is lexically licensed but structurally construed. The Binary_RStage subevent refers to the complex result state (Result Stage ; cf. Caudal 1999b) attached to a transition such as eat. Its binary structure expresses a change-of-state. I will now consider some difficulties related to lexical polysemy and verb-argument relationships.

\subsection{Lexical polysemy and incrementality}

I assume here that the incrementality functions $\mathrm{i}$-inc $/ \mathrm{m}$-inc are lexically specified. Yet the full story is a lot more complicated. Much data suggests that those functions can be modified or determined (when they are lexically underspecified) in context. An overview of a number of problems and a tentative treatment within GL will be proposed here.

\subsubsection{Co-composition and incrementality}

The machinery proposed above is not sufficient to account for subtle cases of lexical polysemy originating in the interaction between the verb and its arguments. Some data would be best treated in terms of co-compostion within $\mathrm{GL}^{5}$ :

5 Roughly, co-composition refers to cases of lexical polysemy in which a lexical item receives a 'new' 
(18a)

* Le moteur acheva de produire un bruit étrange.

The engine finished emitting a strange noise.

(18b) Yannig acheva de produire son article. Yannig finished writing his paper.

The French verb produire yields an i-incremental reading in (18a), vs. a $\mathrm{m}$-incremental reading in (18b). Arguably, produire means 'to cause to come into existence', and therefore makes use of the content of the AGENTIVE qualia role (i.e., the qualia role indicating how a type is brought into existence) of its internal argument to determine the corresponding 'creation' event. The AGENTIVE roles of bruit and article can be represented as follows :
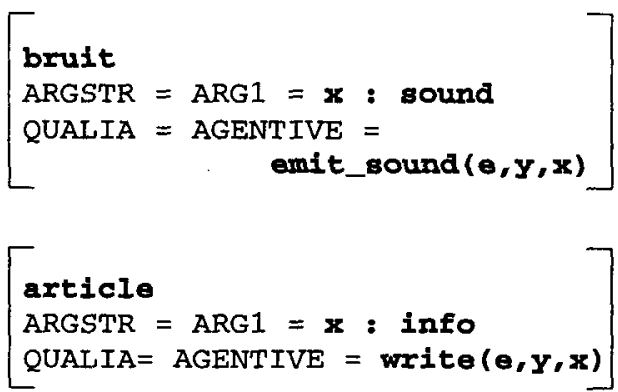

By virtue of the co-composition operation involving events specified in the AGENTIVE of bruit and article, produire interacts differently with its internal argument, and receives different event structures. The omit_sound $(\theta, y, x)$ event in (19) comes along an 1 -inc function mapping the internal argument $x$ onto $e$, while the write $(\theta, y, x)$ event in (20) comes along an $m$-inc function mapping $x$ onto $e$. In fact, the whole event structure of those AGENTIVE roles together with their incrementality functions override those lexically specified by default for produire.

Another limit of GL until recent work (cf. Asher and Pustejovsky 1999) was its inability to construe more versatile qualia role information. Consider the following case of co-composition :

sense (i.e., one not considered to be lexicalized) through the contribution of another lexical item with which it combines. See Pustejovsky (1995). (21a) Yannig finished hiding the bike.

(21b) *Yannig finished hiding the truth.

Hide $x$ arguably means 'to remove $\mathrm{x}$ from accessibility', and obviously the notion of 'accessibility' diverges when $x$ is a physical object (21a) or a proposition (21b). This kind of phenomenological information might be encoded in the FORMAL role for the corresponding super-types and triggered in this context, but a detailed implementation still has to be worked out. See Asher and Pustejovsky (1999) for a discussion of such issues.

\subsubsection{Other cases of polysemy}

Last but not least, many cases of apparent polysemy in the incrementality functions actually arise from the coercion of affected arguments :

(22a) Yannig a fini de ranger sa chambre. Yannig finished tidying up his room.

* Yannig a fini de ranger son livre. (gradual scenarios being left aside) Yannig finished putting away his book.

Ranger receives an incremental reading with chambre in (22a), and no incremental reading in (22b), so that it seems to be properly neither i-incremental nor $\mathrm{m}$-incremental. The way out of this puzzle is the following : ranger is lexically encoded as capable of i-incrementality but not of $m$-incrementality, and the aspectual polysemy of ranger sa chambre originates in the polysemy of chambre. Although there is no question that chambre normally refers to an individual, its meaning is coerced into a collective one in (22a). More precisely, chambre is coerced from an individual real estate sense (immovable_phys_obj) to a collection sense involving the individual objects possibly enclosed within a room (movable_phys_obj), since only the latter is compatible with ranger. One way of accounting for such coercions within GL would be to associate with the CONST qualia role of chambre such a collection of instances of the movable_phys_obj type, the CONST role describing the meronymic constitution of a type. 
In fact, the ability to trigger this very kind of coercion seems to be a general property of verbs addressing their arguments through their FORMAL role (i.e., requiring natural types centrally defined through their CONST and FORMAL - and not functional types - centrally defined through their AGENTIVE and TELIC ; see Pustejovsky 1999). Such verbs are usually able to access their arguments' semantics as individuals through their FORMAL role, and as collections of individuals through their CONST role, if the FORMAL individual does not meet the selectional restrictions imposed by the verb, or other semantic constraints. See Caudal (1998) for detailed evidence of this, and for a tentative solution within GL to the problems raised by the polysemy of collective nouns (e.g., regiment, police and forest), which exhibit a similar behaviour, i.e., can either refer to individuals or to collections. Finally, it should be noted that homeomeronymic nouns (i.e., whose parts and whole refer to the same lexical type, e.g. estate or property seen as land surfaces, or quantity of matter nouns, such as gold or milk; see Winston et al. (1987)) offer other interesting properties w.r.t. to incrementality/atomicity. I will not discuss them here for want of space.

To put it in a nutshell, even prima facie individual-referring nouns such as chambre can behave like collection-referring ones under certain circumstances, making i-incremental readings of normally atomic events possible. Let us move now to some concluding remarks about non-incremental non-atomic events.

\section{On the formal treatment of non- incremental non-atomic events}

I have shown above that the notion of incrementality fell short of explaining the nonatomicity of (13), (14), and (15). I will suggest here a solution based on an extended conception of result states.

The non-incremental, non-atomic events discussed in 3.3 seem to fall into at least two distinct subclasses : scalar events (cf. (13)/(14)) vs. "gradual scenario" events (cf. (15)). I will focus on the former class, the latter class originating clearly in a pragmatic phenomenon ${ }^{6}$. It should be noted that many resultative constructions (e.g., pound the metal flat; see Levin and Rappaport 1995) also receive scalar readings, making the phenomenon a fairly widespread one.

It is a fact that the notions of affectedness and incrementality / event-object mapping do not apply to scalar events. Affectedness indicates that an argument undergoes an incremental (cf. eat) or a definite change of state (cf. throw), and not a gradual but total one, as in the case of scalar verbs (their delimiting arguments are gradually changing as a whole, and not bit by bit). (14) is telic and non-atomic because the chicken goes through successive states of 'cookedness' (i.e., result states) before reaching a final state, and not because of some eventobject mapping function in the spirit of Krifka (1992). Therefore, the telicity of scalar events can only be explained by reference to this scalar change of state, which entails itself a scalar result state. Encoding a richer information about result states in the lexical entries of such verbs, as proposed in Caudal (1999a/b), would allow us to account elegantly for this kind of non-atomic, non-incremental, telic readings of events.

This new conception of result states provide us with a unified account ${ }^{7}$ of (non)-atomicity, incrementality and telicity - a result which generalized paths cannot achieve for reasons exposed above, and others not discussed here. Indeed, even the non-incremental, non-atomic events studied in 3.3 (except (15), but then again this is a pragmatic issue) can also be accounted for in this manner, and path-argument verbs can also be analysed in terms of result states if changes of location undergone by arguments are treated as changes-of-state.

6 Note that contrary to scalar events and incremental events, "gradual scenarios" do not combine with the progressive perfect, cf. *John has been registering his son at the university. This fact suggests that they should be set apart from other non-atomic events, and possibly receive subevents of a different kind.

${ }^{7}$ See Caudal (1999b), where incremental vs. scalar RStages are introduced. 


\section{Conclusion}

It has been demonstrated in this paper that the so-called punctuality of achievements should be reduced to the notion of atomicity. Formal means to calculate it within an NLP system have been discussed; see White (1994) for a computational implementation of related interest, in a similar spirit. The machinery exposed above can be used to predict whether an event should be considered as an accomplishment (non-atomic event; possesses subevents) or an achievement (atomic event; lacks any subevent).

The above developments revealed that (non-)atomicity is at least partly amenable to a compositional semantic procedure, and does not fall altogether under the scope of pragmatics. It has been shown to be directly related to incrementality in many cases, though not in all cases. In order to construe incremental nonatomic events, I proposed to encode $\mathrm{m}$-incrementality vs. i-incrementality in the lexicon, before discussing the accessibility of the internal structure of delimiting argument NPs ; I suggested a solution to the problems raised by the polysemous internal structure of certain nouns. Finally, a tentative result-state based account of non-incremental non-atomic events has been proposed. I even claimed that it can explain all types of non-atomicity and even incrementality in a unified way, and therefore might surpass all the existing accounts of event structure.

\section{References}

Asher, N. and J. Pustejovsky (1999) The Metaphysics of Words in Context. Ms., Brandeis University.

Caudal, P. (1998) Using Complex Lexical Types to Model the Polysemy of Collective Nouns within the Generative Lexicon. Proceedings of DEXA 98, IEEE Computer Society, Los Alamitos, pp. 154160.

Caudal, P. (1999a) Resultativity in French - A Study in Contrastive Linguistics. Paper presented at the $29^{\text {th }}$ Linguistic Symposium on Romance Languages, University of Michigan, Ann Arbor, MI, April.

Caudal, P. (1999b) Result Stages and the Lexicon : The Proper Treatment of Event Structure.
Proceedings of the $9^{\text {th }}$ Conference of the European Chapter of the Association for Computational Linguistics, Bergen, Norway, June.

Declerck, R. (1979). Aspect and the bounded/unbounded (telic/atelic) distinction. Linguistics 17, pp. 761-794.

Dowty, D. (1986) The Effects of Aspectual Class on the Temporal Structure of Discourse : Semantics or Pragmatics? Linguistics and Philosophy, 9, pp. 3761.

Dowty, D. (1991) Thematic Proto-Roles and Argument Selection. Languages 67/3, pp. 547-619.

Jackendoff, R. (1996) The Proper Treatment of Measuring Out, Telicity and Perhaps Event Quantification in English. Natural Language and Linguistic Theory, 14, pp. 305-354.

Krifka, M. (1992) Thematic Relations as Links between Nominal Reference and Temporal Constitution. In Lexical Matters, I. Sag and A. Szabolsci, eds., CSLI, Stanford, CA, pp. 29-53.

Levin, B. and M. Rappaport Hovav (1995) Unaccusativity: At the Syntax - Lexical Semantics Interface. MIT Press, Cambridge, MA.

Link, G. (1983) The Logical Analysis of Plurals and Mass Terms. in R. Baüerle, C. Schwarze and A. von Stechow (eds.), Meaning, Use and Interpretation of Language, Walter de Gruyter, Berlin, pp. 302-323.

Moens, M. and M. Steedman (1988) Temporal Ontology and Temporal Reference. Computational Linguistics, 14/2, pp.15-28.

Pustejovsky, J. (1995) The Generative Lexicon. MIT Press, Cambridge, MA.

Pustejovsky, J. (1999) Decomposition and Type Construction. Ms., Brandeis University.

Tenny, C. (1994) Aspectual Roles and the SyntaxSemantics Interface, Kluwer, Dordrecht.

Vendler, Z. (1957) Verbs and Times. The Philosophical Review, 66, pp. 143-160.

Verkuyl, H. (1993) A Theory of Aspectuality. Cambridge University Press, Cambridge.

Winston, M.E., R. Chaffin and D. Hermann (1987) A taxonomy of part-whole relations. Cognitive Science, 11, pp. 417-444.

White, M. (1994) A Computational Approach to Aspectual Composition. Unpublished Ph.D. dissertation, Institute for Research in Cognitive Science, University of Pennsylvania, Philadelphia.

\section{Acknowledgements}

Many thanks to James Pustejovsky for the very fruitful discussions we had about incrementality. 